\title{
Anti streptolysin O (ASO) titers in normal healthy children aged between 5 to 15 years in Ujjain region
}

\author{
Madaan $\mathbf{R}^{1}$, Mandliya $\mathbf{J}^{2}$, Tiwari $\mathbf{H}^{3}$, Dhaneria $\mathbf{M}^{4}$, Gupta $\mathbf{R}^{5}$, Pathak $\mathrm{A}^{6}$ \\ ${ }^{1}$ Dr. Rahul Madaan, ${ }^{2}$ Dr. Jagdish Mandliya, ${ }^{3}$ Dr. H L Tiwari, ${ }^{4}$ Dr. Mamta Dhaneria, Department of Pediatrics, R. D. \\ Gardi Medical College, Ujjain, M.P, India, ${ }^{5}$ Dr. Rajesh Gupta, Department of Pediatrics, Chirayu Medical College \& \\ Hospital, Bhopal, M.P. India, ${ }^{6}$ Dr. Ashish Pathak, Department of Women and Children's Health, International Maternal \\ and Child Health Unit, Uppsala University, Uppsala, Sweden.
}

Address for Correspondence: Dr Rahul Madaan, Madaan Nursing Home, Opposite Zila Parishad, GT Road, Bathinda (Punjab), e-mail: rahulmadaan52@gmail.com

\begin{abstract}
Introduction: Rheumatic fever is an inflammatory disease that may develop after an infection with Streptococcus pyogenes bacteria, believed to be caused by antibody cross reactivity that can involve heart, joints, skin and brain. Measurement of Anti Streptolysin O (ASO) antibodies to specific streptococcal antigens is therefore necessary for the diagnosis of the preceding Group A Streptococcal (GAS) infection. Aims and objectives: To determine the upper limit of the normal ASO titers in normal healthy school going children aged 5-15 years and to determined a baseline value to compare with when a single ASO titer is available. Material and method: A community based cross sectional study was done on normal healthy children aged 5-15 years divided into group 1 (5-10 years) and group 2 (11-15 years) after taking informed consent from parents. Blood sample were collected after thorough sterilization of the area. ASO testing from serum sample was done using TURBILYTE Antistreptolysin ' $O$ ' diagnostic reagent for quantitative in-vitro determination of ASO in serum on photometric systems. Results: Out of total 200 children included in the study, 100 were in group 1 and 100 in group 2, $118(59 \%)$ were males and $82(41 \%)$ were females. The mean ASO titer for group 1 was $105.69 \mathrm{IU}$ and that of group 2 was $144.73 \mathrm{IU}$ with a standard deviation of 3.675 and 5.823 respectively ( $P$ value $<0.05$ ). Out of total 200 students 160 were living in overcrowded conditions. The mean ASO titer of children with overcrowded living conditions was $131.41 \mathrm{IU}$ with a standard deviation of 53.472, and those with non-overcrowded living condition was 100.42 IU with a standard deviation of 39.30 ( $P$ value $<0.05)$. Conclusion: The upper limit of normal (ULN) was greater in the children of Group 1 ( $P$ value $<0.05,200 \mathrm{Vs} 135)$. No statistically significant difference was found in the ASO titer according to gender. Statistically significant high mean ASO titer (131.41 IU) was found in children living in overcrowded conditions as compared to those living under non-overcrowded conditions (100.41 IU).
\end{abstract}

Keywords: Antistreptolysin O, Streptococcus pyogenes, Rheumatic fever, Upper limit of normal

\section{Introduction}

Streptococcus pyogenes or Lancefield Group A betahemolytic streptococcus (GAS), is one of the commonest bacterial pathogens that causes acute pharyngitis among school-aged children living in lower socioeconomic conditions [1]. Acute rheumatic fever is an inflammatory disease of the heart, joints, central nervous system subcutaneous tissues that develops after a nasopharyngeal infection by one of the group A beta hemolytic streptococci [2]. Rheumatic heart disease (RHD) is the second most frequent form of acquired

Manuscript received: $24^{\text {th }}$ August 2015

Reviewed: $2^{\text {nd }}$ September 2015

Author Corrected: $10^{\text {th }}$ September 2015

Accepted for Publication: $16^{\text {th }}$ September 2015 heart disease in children worldwide and almost all cases and deaths occur in developing countries [3]. Group A streptococcal (GAS) infection and their sequelae like acute rheumatic fever and RHD are important and major health problem in India [4]. An absolute requirement for the diagnosis of acute rheumatic fever is supporting evidence of a recent GAS infection. One third of patients with acute rheumatic fever have no history of an antecedent pharyngitis.

Therefore, evidence of an antecedent GAS infection is usually based on elevated or increasing serum Anti streptococcal antibody (ASO) titers [5]. The antibody 
produced by the human host against this toxin, ASO, is the most widely used and the most standardized of the group A streptococcal antibody tests available [6]. ASO titer has been shown to vary with geographical location, age, season and site of infection. Hence, when ASO titer from single specimen is available it is compared with the predetermined baseline values in a given geographical area [7]. Rapid, quantitative turbidimetric immunoassay for serum ASO has proven to be superior to other available methods for measuring serum ASO [8].

The incidence of rheumatic fever varies from $0.2-0.75$ per 1000 children of 5-15 years age group whereas in India it is estimated to be $1-5.04$ per 1000 cases [4]. Such data have emphasized the importance of accurate clinical diagnosis, often requiring laboratory confirmation of preceding GAS infection. It is not always possible to obtain clinical history or to recover the organism moreover it is not feasible to obtain acute and convalescent sera. Positive throat culture are obtained only in about $11 \%$ at the time of presentation of acute rheumatic fever, moreover mere presence of organism in the throat can also indicate carrier state which is seen in $2.5-35.4 \%$ of the individuals. In such cases the presence of a host immune response is the only evidence of the recent infection that remains. Measurement of antibodies to specific streptococcal antigens is therefore necessary for the diagnosis of the preceding GAS infection. ASO levels rise rapidly after about 3-4 weeks post streptococcal infection and remains elevated for months. This study was planned to determine the upper limit of the normal (ULN) of the ASO titers in normal healthy school going children aged 5-15 years; to determine the role of ASO titers in the early diagnosis of antecedent infections caused by group A beta hemolytic streptococcus like acute rheumatic fever and post streptococcal glomerulonephritis and to determined a baseline value to compare with when a single ASO titer is available.

\section{Material and Methods}

This community based cross sectional study was done involving the students of residential school Palwa and Balakheda, District Ujjain, Madhya Pradesh during The period of November 2012 to February 2013 after written informed consent was obtained from the principal of the schools and from the parents of children. The data was collected in a predesigned and pretested proforma and contained epidemiological and clinical determinants required according to the objectives of the study. Normal healthy children belonging to 5-15 years of age group were included in the study. Children with recent history of sore throat, fever, dark colored urine, rash, joint pains were excluded.

For ASO testing, collection of blood was done under aseptic precautions. Two $\mathrm{ml}$ of blood was collected and serum separated after allowing the blood to clot. The test was done by using TURBILYTE Antistreptolysin ' $\mathrm{O}$ ' diagnostic reagent - for quantitative in vitro determination of ASO in serum on photometric systems. The ASO kit was manufactured by TULIP Diagnostic Systems VOLMOLENHEIDE 13 B-2400 MOL Belgium. Data was entered in Epi Data 3.1 software and appropriate statistical methods were used for data analysis using STATA 10.0 (Stata Corp., College Station, TX, USA) statistical software.

The independent sample t-test was used for comparison of continuous (numerical) variables, after checking for a normal distribution. The Chi-square test was used for comparison of categorical values, P-value $<0.05$ were considered significant.

Prior to conduction of this study, ethical approval was obtained from Institutional and Ethics Committee, R D gardi Medical College Ujjain.

\section{Results}

A total of 200 students were studied and divided into two age groups, group 1 (5-10 years) and group 2 (11-15 years) each group having 100 children. A total of 118 (59\%) students were males and $82(41 \%)$ were females. The group wise distribution of sex showed that 49 (41.5\%) males belonged to age group 1 and $69(58.5 \%)$ males belonged to age group 2; while $51(62.2 \%)$ females belonged to age group 1, 31 (37.8\%) females belonged to age group 2 (Table 1 and Figure 1). Children living in over crowded house were $160(80 \%)$. Out of these $64(40 \%)$ children belonged to age group 1 and $96(60 \%)$ children belonged to age group 2. The mean ASO titer for group 1 was 105.69 and that of group 2 was 144.73 with a standard deviation of 3.675 and 5.823, respectively. There was a significant difference in the two groups ( $P$ value $<0.05$ ). Maximum percentage of the population in both the sexes were having ASO titers on the lower side (less than 150 IU) and almost equal percentage of the population in both the sexes had ASO titers on the higher side (More than 200 IU) (Table 2). The mean ASO titer of those having over crowded living condition was 131.41 with a standard deviation of 
53.472, and those with non-overcrowded living condition was 100.42 with a standard deviation of 39.30 . The difference was statistically significant $(P<0.05)$ (Table 4$)$.

Table-1: Shows the distribution of ASO titers in 200 children distributed in two groups (n=100), group 1 aged 5 to 10 years and group 2 aged 11 to 15 years.

\begin{tabular}{|c|c|c|c|}
\hline $\begin{array}{c}\text { ASO titers } \\
(\mathbf{I U})\end{array}$ & Group 1(5-10 years) & Group 2(11-15 years) & Total (n= 200) \\
\hline$<\mathbf{1 0 0}$ & $59(69.4 \%)$ & $26(30.6 \%)$ & $85(42.5 \%)$ \\
\hline $\mathbf{1 0 0 - 1 2 4}$ & $12(52.1 \%)$ & $11(47.9 \%)$ & $23(11.5 \%)$ \\
\hline $\mathbf{1 2 5}-149$ & $12(54.5 \%)$ & $10(45.5 \%)$ & $22(11 \%)$ \\
\hline $\mathbf{1 5 0 - 1 7 4}$ & $13(43.3 \%)$ & $17(56.7 \%)$ & $30(15 \%)$ \\
\hline $\mathbf{1 7 5 - 1 9 9}$ & $1(6.2 \%)$ & $15(93.8 \%)$ & $16(8 \%)$ \\
\hline $\mathbf{2 0 0 - 2 2 4}$ & $2(12.5 \%)$ & $14(87.5 \%)$ & $8(8 \%)$ \\
\hline $\mathbf{2 2 4 - 2 5 0}$ & $1(12.5 \%)$ & $7(87.5 \%)$ & $\mathbf{2 0 0}$ \\
\hline Total & $\mathbf{1 0 0}$ & $\mathbf{1 0 0}$ & \\
\hline
\end{tabular}

Table-2: Shows the distribution of ASO titers in $\mathbf{2 0 0}$ children distributed according to male and female sex.

\begin{tabular}{|c|c|c|c|}
\hline & \multicolumn{2}{|c|}{ Sex } & \multirow{2}{*}{ Total } \\
\cline { 1 - 3 } ASO titer (IU) & Male & Female & $85(42.5 \%)$ \\
\hline$<\mathbf{1 0 0}$ & $41(48.2 \%)$ & $44(51.8 \%)$ & $23(11.5 \%)$ \\
\hline $\mathbf{1 0 0 - 1 2 4}$ & $16(69.5 \%)$ & $5(30.5 \%)$ & $22(11 \%)$ \\
\hline $\mathbf{1 2 5 - 1 4 9}$ & $17(77.2 \%)$ & $6(22.8 \%)$ & $30(15 \%)$ \\
\hline $\mathbf{1 5 0 - 1 7 4}$ & $24(80 \%)$ & $8(50 \%)$ & $16(8 \%)$ \\
\hline $\mathbf{1 7 5 - 1 9 9}$ & $8(50 \%)$ & $9(56.3 \%)$ & $16(8 \%)$ \\
\hline $\mathbf{2 0 0 - 2 2 4}$ & $7(43.7 \%)$ & $3(37.5 \%)$ & $8(4 \%)$ \\
\hline $\mathbf{2 2 5 - 2 5 0}$ & $5(62.5 \%)$ & $\mathbf{8 2}(\mathbf{4 1} \%)$ & $\mathbf{2 0 0}(\mathbf{1 0 0} \%)$ \\
\hline Total & $\mathbf{1 1 8 ( 5 9 \% )}$ & & \\
\hline
\end{tabular}

Table-3: Shows the distribution of ASO titers in 200 children distributed according to presence or absence of overcrowding.

\begin{tabular}{|c|c|c|c|}
\hline \multirow{2}{*}{ ASO titer } & \multicolumn{2}{|c|}{ Living condition } & \multirow{2}{*}{ Total (n=200) } \\
\cline { 2 - 4 } & Overcrowding & No overcrowding & $85(42.5 \%)$ \\
\hline$<\mathbf{1 0 0}$ & $58(68.2 \%)$ & $27(31.2 \%)$ & $23(11.5 \%)$ \\
\hline $\mathbf{1 0 0 - 1 2 4}$ & $18(78.2 \%)$ & $5(21.2 \%)$ & $22(11 \%)$ \\
\hline $\mathbf{1 2 5}-149$ & $19(86.3 \%)$ & $3(13.7 \%)$ & $30(15 \%)$ \\
\hline $\mathbf{1 5 0 - 1 7 4}$ & $27(90 \%)$ & $3(10 \%)$ & $16(8 \%)$ \\
\hline $\mathbf{1 7 5 - 1 9 9}$ & $16(100 \%)$ & $0(0 \%)$ & $16(8 \%)$ \\
\hline $\mathbf{2 0 0 - 2 2 4}$ & $14(87.5 \%)$ & $2(12.5 \%)$ & $8(4 \%)$ \\
\hline $\mathbf{2 2 5 - 2 5 0}$ & $8(100 \%)$ & $0(0 \%)$ & $\mathbf{2 0 0}(\mathbf{1 0 0} \%)$ \\
\hline Total & $\mathbf{1 6 0}(\mathbf{8 0} \%)$ & $\mathbf{4 0}(\mathbf{2 0} \%)$ & \multirow{2}{*}{} \\
\hline
\end{tabular}


Original Research Article

Table-4: Shows the distribution of mean values, standard deviation and standard error of ASO titers in 200 children distributed according to presence or absence of overcrowding.

\begin{tabular}{|c|c|c|c|c|c|c|c|}
\hline \multirow{2}{*}{ ASO } & Living & $\mathbf{n = 2 0 0}$ & Mean & Std. Deviation & Std. Error & T & P \\
\cline { 2 - 8 } & Overcrowding & 160 & 131.41 & 53.472 & 4.227 & 3.43 & 0.001 \\
\cline { 2 - 8 } & No overcrowding & 40 & 100.42 & 39.300 & 6.214 & & \\
\hline
\end{tabular}

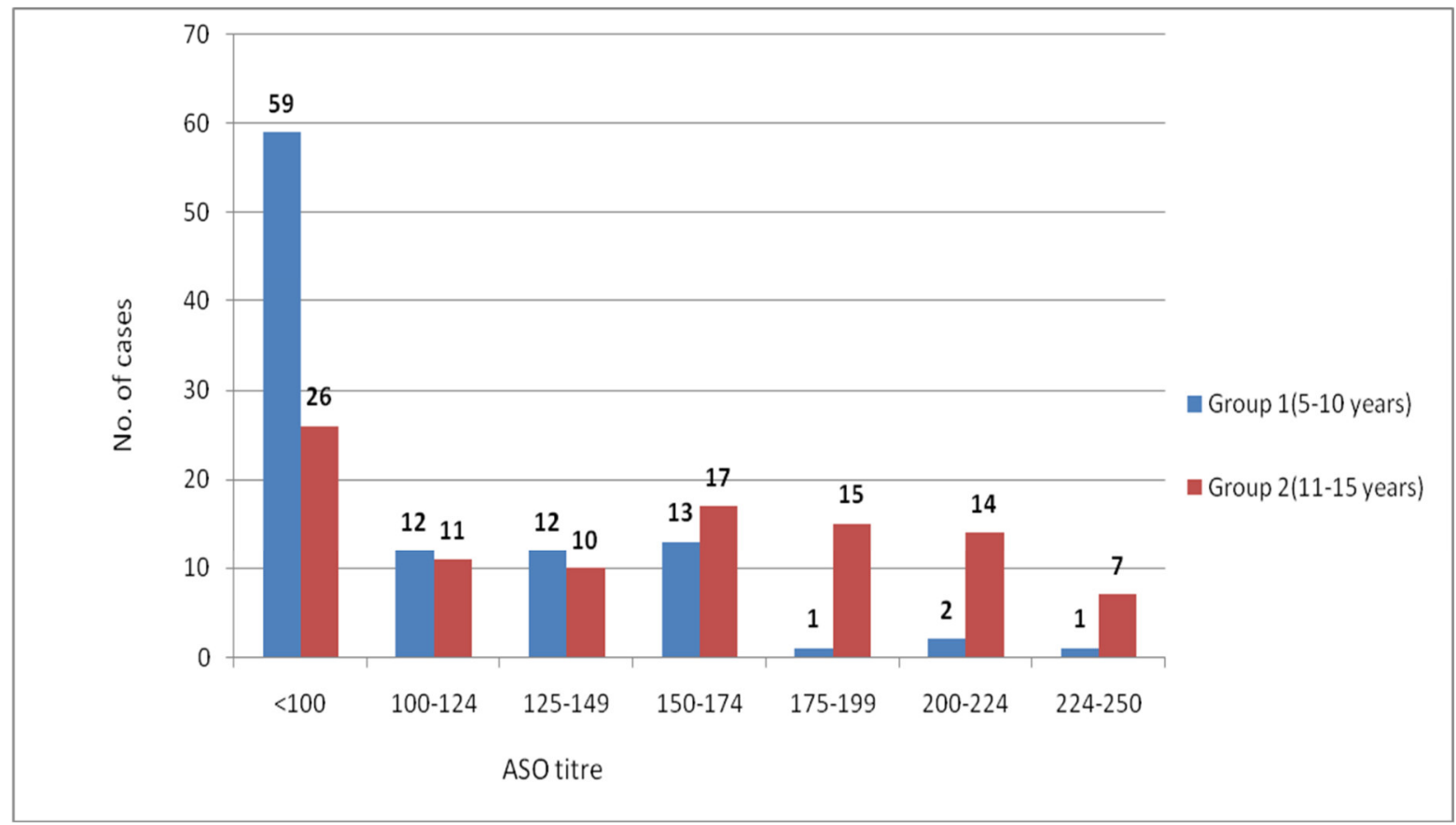

Figure-1: Bar chart showing number of children belonging to the two age groups distributed according to the ASO titers.

\section{Discussion}

An absolute requirement for the diagnosis of acute rheumatic fever is the supporting evidence of group A streptococcal infection. Evidence of antecedent group A streptococcal infection is usually based on elevated or rising serum ASO antibody titers.

Antibody titers may be elevated in the absence of clinical or bacteriological evidence of streptococcal pharyngitis. The ASO titer is the most popular antibody test. The normal level of ASO titer is defined as the highest titer exceeded by only $20 \%$ of the population. ULN is defined as that titer exceeded by 20 percent of a normal population [8]. The geometric mean titer and ULN for the ASO for the western world was determined to be 120 IU [9]. It is influenced significantly by age, geography, season and other factors. ASO titers of more than 333 are generally considered elevated in children however this was found to be $170 \mathrm{IU}$ in our study. The geometric mean titer (GMT) and upper limit of normal
(ULN) in our study group was greater in the children of age group $5-10$ years (which was statistically highly significant, i.e. $p$ value $<0.05,200$ vs. 135) than the standard ASO titer quoted -250 IU for the diagnosis of acute rheumatic fever. The ULN of ASO titers in Mysore is determined to be 242 IU [7], 239 IU in Chandigarh and 305 IU in Mumbai [10]. Our study group did not have any history of repeated sore throat infections and fever. No difference was found in the ASO titer according to gender. The mean value of ASO titers in males was $128.08 \mathrm{IU}$ and in the females was 121.07 IU and the difference was not statistically significant $(\mathrm{p}=0.353)$.

Our study also showed a high mean ASO titer (131.41 IU) in children living in overcrowded conditions as compared to those living under normal conditions (100.41 IU) and this difference was statistically significant $(\mathrm{p}<0.05)$. 
Original Research Article

\section{Conclusion}

Having established the upper limit of normal in school age children in our population, we can consider this as the baseline ASO titer (170 IU). This would help in interpreting the ASO titer in suspected acute rheumatic fever patients in population of Ujjain region. This value of $170 \mathrm{IU}$ is representative of our school going population and would immensely help the Pediatricians, Epidemiologists and the Microbiologists to interpret streptococcal antibody titer correctly.

Acknowledgement:Authors would like to acknowledge Dr. J K Sharma, Dean, R D Gardi Medical College Ujjain and Dr. V K Mahadik, Director, R D Gardi Medical College Ujjain for granting permission and providing financial assistance for conduction of this study.

Funding: Nil, Conflict of interest: None initiated, Perission from IRB: Yes

\section{References}

1. Bisno AL. Acute pharyngitis: etiology and diagnosis. Pediatrics. 1996 Jun;97(6 Pt 2):949-54.

2. Lennon D. Acute Rheumatic Fever. In: Feigin R and CherryJ(Eds),Textbook of Pediatric Infectious Diseases, $5^{\text {th }}$ ed. Philadelphia: WB Saunders; 2004:413-26.

3. Carapetis JR. A review of WHO activities. In: The Burden of and the Evidence for Strategies to Control Group A Streptococcal Diseases: Part 3: The Current Evidence for the Burden of Group A Streptococcal Diseases.Geneva:World Health Organization;2004: $1-49$.

4. Sethi S, Kaushik K, Mohandas K, Sengupta C, Singh $\mathrm{S}$, Sharma M. Anti-streptolysin O titers in normal healthy children of 5-15 years. Indian Pediatr. 2003 Nov;40(11):1068-71.

5. Stollerman GH. Rheumatic fever. Lancet. 1997 Mar 29;349(9056):935-42.

6. Kaplan EL, Ferrieri P, Wannamaker LW. Comparison of the antibody response to streptococcal cellular and extracellular antigens in acute pharyngitis. $\mathrm{J}$ Pediatr. 1974 Jan; 84(1):21-8.

7. Mahendrappa Kotyal B, Rajendra. Upper Limit of Normal Antistreptolysin-O Titer in Healthy School Children. Indian Pediatr. 2010 July; 47(7): 629.

8. Shogo Otsuji, TetsuroKamada, Tsuneaki Matsuura, Masashi Seki, Kiko Tanaka, Hideaki Shibata and Tetsuro Honda. A rapid turbidimetric immunoassay for serum antistreptolysin- $\mathrm{O}_{2}$. Journal of Clinical Laboratory Analysis.2005: 241-245. DOI: 10. 1002/jcla. 1860040402.

9. WANNAMAKER LW, AYOUB EM. Antibody titers in acute rheumatic fever. Circulation. 1960 Apr; 21:598-614.

10. Danchin MH, Carlin JB, Devenish W, Nolan TM, Carapetis JR. New normal ranges of antistreptolysin O and antideoxyribonuclease B titres for Australian children. J Paediatr Child Health. 2005 Nov;41 (11):583-6.

11. MG Karmarkar, Vineetha Venugopal, Leela Joshi \& Riecha Kamboj. Evaluation and revaluation of upper limits of normal values of anti- Streptolysin O and anti deoxyribonucleasse B in Mumbai. Indian J Med Res. 2004;119:26-28.

\section{How to cite this article?}

Madaan R, Mandliya J, Tiwari H L, Dhaneria M, Gupta R, Pathak A. Anti streptolysin O (ASO) titers in normal healthy children aged between 5 to 15 years in Ujjain region. J PediatrRes.2017;4(02):120-124.doi:10.17511/ijpr.2017.i02.05. 